DOI 10.18699/SBB-2020-10

\title{
Structural heterogeneity of the EIN3 binding site affects the characteristics of transcriptional response to ethylene in Arabidopsis
}

\author{
Dolgikh V.A. ${ }^{1 *}$, Levitsky V.G. ${ }^{1,2}$, Oschepkov D.Y. ${ }^{1}$, Zemlyanskaya E.V. ${ }^{1,2}$ \\ ${ }^{1}$ Institute of Cytology and Genetics SB RAS, Novosibirsk, Russia \\ 2. Novosibirsk State University \\ *e-mail:dolgikh@bionet.nsc.ru
}

Key words: bioinformatics, transcription factor, ETHYLENE-INSENSITIVE3, EIN3 binding site, ChIP-seq, RNA-seq

Motivation and Aim: ETHYLENE INSENSITIVE 3 (EIN3) transcription factor is the key transcriptional regulator of ethylene response. It activates target genes transcription upon binding a short sequence in their promoters called EBS (EIN3 binding site) [1]. EIN3 is able to bind DNA as a homodimer and as a heterodimer [2]. It is possible, the nucleotide context at the flanks can determine the protein binding mode forming EBS structural variants. In turn, a variety of structural variants can provide variability of ethylene transcriptional response. However, genome-wide studies of the EBS architecture have not been conducted. Here we performed a systematic bioinformatics analysis of the EIN3 binding site architecture and its role in the regulation of the EIN3-dependent transcriptional response to ethylene.

Methods and Algorithms: We analyzed publicly available ChIP-seq data on EIN3 binding and RNA-seq data on ethylene-induced transcriptomes for 1, 4 and 12 hours of ethylene treatment [3]. For motif analysis, we used Homer [4] and MCOT [5]. To associate EBS structural variants with EIN3 binding and response to ethylene we performed Fisher's exact test.

Results: We discovered three structural variants of EBS in EIN3 ChIP-seq peaks. (1) 2EBS(-1), a head-tohead EBS repeat with 1 bp overlap; (2) GC-EBS, monomeric EBS flanked with GC-rich tract upstream the core EBS; (3) TEIL-like EBS, monomeric structural variant similar to the binding site of EIN3 homolog in tobacco. We found genes containing each of them in promoter. Using EIN3 ChIP-seq binding data, we found that only $2 \mathrm{EBS}(-1)$ was stably bound with EIN3. Using ethylene-induced RNA-seq data we found that of all structural variants only 2EBS(-1) was strongly associated with the ethylene response.

Conclusion: The results obtained indicate that 2EBS(-1) is the main structural variant of EBS, providing the most pronounced response to ethylene. Whereas GC-EBS and TEIL-like EBS can mediate more specific ethylene-related reactions.

Acknowledgements: Supported by the RSF (20-14-00140) and State Budget Project (0324-2019-0040).

\section{References}

1. Kosugi S. and Ohashi Y. (2000) Cloning and DNA-binding properties of a tobacco Ethylene-Insensitive3 (EIN3) homolog. Nucleic Acids Res. 28(4):960-967.

2. Song J. et al. (2015) Biochemical and Structural Insights into the Mechanism of DNA Recognition by Arabidopsis ETHYLENE INSENSITIVE3. PLoS One. 10(9):e0137439.

3. Chang K.N. et al. (2013) Temporal transcriptional response to ethylene gas drives growth hormone cross-regulation in Arabidopsis. Elife. 2:e0675.

4. Heinz S. et al. (2010) Simple combinations of lineage-determining transcription factors prime cis-regulatory elements required for macrophage and b cell identities. Mol. Cell. 38(4):576-589.

5. Levitsky V. et al. (2019) A single ChIP-seq dataset is sufficient for comprehensive analysis of motifs co-occurrence with MCOT package. Nucleic Acids Res. 47(21):e139. 